\title{
Genome-wide identification and expression analysis of the calmodulin-binding transcription activator (CAMTA) gene family in wheat (Triticum aestivum L.)
}

Fan Yang ${ }^{1 \dagger}$, Fu-shuang Dong ${ }^{1 \dagger}$, Fang-hui Hu ${ }^{2}$, Yong-wei Liu', Jian-fang Chai ${ }^{1}$, He Zhao ${ }^{1}$, Meng-yu Lv ${ }^{1}$ and Shuo Zhou ${ }^{1 *}$ iD

\begin{abstract}
Background: Plant calmodulin-binding transcription activator (CAMTA) proteins play important roles in hormone signal transduction, developmental regulation, and environmental stress tolerance. However, in wheat, the CAMTA gene family has not been systematically characterized.

Results: In this work, 15 wheat CAMTA genes were identified using a genome-wide search method. Their chromosome location, physicochemical properties, subcellular localization, gene structure, protein domain, and promoter cis-elements were systematically analyzed. Phylogenetic analysis classified the TaCAMTA genes into three groups (groups A, B, and C), numbered 7, 6, and 2, respectively. The results showed that most TaCAMTA genes contained stress-related cis-elements. Finally, to obtain tissue-specific and stress-responsive candidates, the expression profiles of the TaCAMTAs in various tissues and under biotic and abiotic stresses were investigated. Tissue-specific expression analysis showed that all of the 15 TaCAMTA genes were expressed in multiple tissues with different expression levels, as well as under abiotic stress, the expressions of each TaCAMTA gene could respond to at least one abiotic stress. It also found that 584 genes in wheat genome were predicted to be potential target genes by CAMTA, demonstrating that CAMTA can be widely involved in plant development and growth, as well as coping with stresses.

Conclusions: This work systematically identified the CAMTA gene family in wheat at the whole-genome-wide level, providing important candidates for further functional analysis in developmental regulation and the stress response in wheat.
\end{abstract}

Keywords: CAMTA, Wheat, Genome-wide identification, Gene expression

\footnotetext{
* Correspondence: zhoushuobio@163.com

${ }^{\dagger}$ Fan Yang and Fu-shuang Dong contributed equally to this work. 'Institute of Genetics and Physiology, Hebei Academy of Agriculture and Forestry Sciences/Plant Genetic Engineering Center of Hebei Province, Shijiazhuang 050051, People's Republic of China

Full list of author information is available at the end of the article
}

(c) The Author(s). 2020 Open Access This article is licensed under a Creative Commons Attribution 4.0 International License, which permits use, sharing, adaptation, distribution and reproduction in any medium or format, as long as you give appropriate credit to the original author(s) and the source, provide a link to the Creative Commons licence, and indicate if changes were made. The images or other third party material in this article are included in the article's Creative Commons licence, unless indicated otherwise in a credit line to the material. If material is not included in the article's Creative Commons licence and your intended use is not permitted by statutory regulation or exceeds the permitted use, you will need to obtain permission directly from the copyright holder. To view a copy of this licence, visit http://creativecommons.org/licenses/by/4.0/. The Creative Commons Public Domain Dedication waiver (http://creativecommons.org/publicdomain/zero/1.0/) applies to the data made available in this article, unless otherwise stated in a credit line to the data. 


\section{Background}

$\mathrm{Ca}^{2+}$ signals, one of the most important secondary messengers in plants, are widely involved in many adaptive and developmental processes [1]. In plants, there are three main classes of $\mathrm{Ca}^{2+}$ sensors to decode and transmit the $\mathrm{Ca}^{2+}$ signals, including calmodulin (together with calmodulin-like proteins) (CaMs/CMLs), calcium-dependent protein kinases (CDPKs), and calcineurin B-like proteins (CBLs) [2]. Most of the calmodulin/calmodulin-like proteins execute their biological functions by binding to calmodulin-binding proteins (CaMBPs), including transcription factors, protein kinases, ion channels, and enzymes, with the exception of CaM7, which can act as a transcription factor to directly regulate the expression of the HY5 gene [3]. To our knowledge, plant CaMs can regulate at least 90 transcription factors, including calmodulin-binding transcription activators (CAMTAs) [4].

CAMTA proteins are characterized by several conserved domains, including a unique DNA-binding domain (CG1), a transcription factor immunoglobulin-like DNAbinding domain (TIG), ankyrin repeats (ANK), IQ motifs (IQXXXRGXXXR), and a $\mathrm{Ca}^{2+}$-dependent calmodulinbinding domain (CaMBD) [5-7]. To date, the CAMTA gene family has been identified in a wide variety of plant species, such as Arabidopsis (Arabidopsis thaliana, six members) [7], tomato (Solanum lycopersicum, seven members) [8], rice (Oryza sativa, seven members) [9], grape (Vitis vinifera, 10 members) [10], maize (Zea mays, nine members) [11], soybean (Glycine max, 15 members) [12], rape (Brassica napus, 18 members) [13], alfalfa (Medicago sativa, seven members) [14], poplar (Populus trichocarpa, seven members) [4], and citrus (Citrus sinensis and Citrus clementina, nine members) [15].

CAMTAs have been shown to be extensively involved in plant growth and developmental regulation, as well as in biotic and abiotic stress tolerance. In Arabidopsis, CAMTA1 and CAMTA2 work in concert with CAMT A3 to directly bind to the promoter of C-repeat binding factor2 (CBF2) to induce expression, leading to increased plant freezing tolerance [16, 17]. While AtCAMTA1 also positively regulates drought responses by regulating a few stress-responsive genes, including responsive to dehydration26 (RD26), early response to dehydration7 (ERD7), responsive to ABA18 (RAB18), lipid transfer proteins (LTPS), cold-regulated78 (COR78), $C B F 1$, and heat shock proteins (HSPs) [18], AtCAMTA3 can act as a negative regulator of plant immunity to modulate pathogen defense responses by activating the EDS1-mediated salicylic acid (SA) signaling [19]. A recent study showed that TaCAMTA4 may function as a negative regulator of the defense response against Puccinia triticina, since the virus-induced gene silencing (VIGS)-based knockdown of TaCAMTA4 resulted in the enhanced resistance to $P$. triticina race 165 [20]. This suggested that one CAMTA member usually participates in multiple signaling pathways, while multiple CAMTA members often work together to participate in one signaling pathway.

Here, we obtained 15 TaCAMTA genes from wheat genomes. Their chromosome location, physicochemical properties, subcellular localization, gene structure, protein domain, promoter cis-elements, and expression profiles in multiple tissues as well as in response to stresses were systematically analyzed. Our work has established a foundation for the further analysis of wheat CAMTA genes and provides a basic understanding of their roles in development and stress responses.

\section{Results and discussion}

\section{Identification of the TaCAMTA gene family in wheat}

Using the method described below, a total of 15 TaCAMTA genes were identified in wheat. Since the TaCAMTA genes were clustered into six homoeologous groups, these genes were designated as TaCAMTA1 to TaCAMTA6 according to their homology with rice $C A M T A$ genes, plus a suffix corresponding to the specific wheat genome identifier (A, B, or D) for each gene name (Table 1, Fig. 1). For example, the TaCAMTA1 genes in genomes $\mathrm{A}, \mathrm{B}$, and $\mathrm{D}$ were named TaCAMTA1-A, TaCAMTA1-B, and TaCAMTA1-D, respectively. The results showed that TaCAMTA1, 2, 3, and 4 contained three homolog genes (TaCAMTA1-A/B/D, 2$A / B / D, 3-A / B / D$, and $4-A / B / D)$, while TaCAMTA5 harbored two (TaCAMTA5-A/D), and TaCAMTA6 possessed one (TaCAMTA6-B). The highest number (eight genes: TaCAMTA3- $A / B / D, 4-A / B / D$, and $5-A / D)$ of TaCAMTA genes was found in homoeologous group 2, three TaCAMTA genes (TaCAMTA1-A/B/D) in homoeologous group 3, three TaCAMTA genes (TaCAMTA2-A/B/D) in homoeologous group 4 , and one TaCAMTA gene $($ TaCAMTA6-B) in homoeologous group 5 , while no TaCAMTA gene was identified in homoeologous groups 1,6 , and 7. Information relating to the 15 TaCAMTA genes, including gene names, locus IDs, open reading frame (ORF) lengths, chromosome locations, and the deduced polypeptides is provided in Table 1 . The predicted TaCAMTA proteins contain 805 (TaCAMTA1-B) to 1067 (TaCAMTA2-B) amino acid residues, with molecular weights ranging from $90.82 \mathrm{kDa}$ (TaCAMTA1-B) to $119.32 \mathrm{kDa}$ (TaCAMTA2-A), and the isoelectric points ranged from 5.14 (TaCAMTA4-B) to 8.96 (TaCAMTA5A) (Table 1).

The size of the CAMTA gene family in wheat is similar to that of oilseed rape (B. napus) and soybean (G. max) with 18 and 15 members [12, 13], respectively, but is higher than that of $A$. thaliana with six members, citrus (C. sinensis and C. clementina) with nine members, maize (Z. mays) with nine members, and alfalfa ( $M$. 
Table 1 Information of the 15 CAMTA gene members in wheat

\begin{tabular}{|c|c|c|c|c|c|c|c|c|}
\hline Gene & Locus ID & Chr. location $^{a}$ & $\begin{array}{l}\text { ORF length } \\
\text { (bp) }\end{array}$ & Length (AA) & MW (kDa) & pl & $\begin{array}{l}\text { Subcellular } \\
\text { localization }\end{array}$ & $\begin{array}{l}\text { Ortholgous } \\
\text { genes in rice }\end{array}$ \\
\hline TaCAMTA1-A & TraesCS3A02G433300 & $3 \mathrm{~A}(-): 674751244-674,757,428$ & 3420 & 821 & 92.48 & 7.5232 & Nucleus & OsCAMTA1 \\
\hline TaCAMTA1-B & TraesCS3B02G469100 & $3 B(-): 715886740-715,893,072$ & 3253 & 805 & 90.82 & 7.1271 & Nucleus & \\
\hline TaCAMTA1-D & TraesCS3D02G426700 & $3 D(-): 539903862-539,910,357$ & 3499 & 818 & 92.51 & 7.2482 & Nucleus & \\
\hline TaCAMTA2-A & TraesCS4A02G407100 & $4 A(-): 679981642-679,990,156$ & 3806 & 1066 & 119.32 & 5.8827 & Nucleus & OsCAMTA2 \\
\hline TaCAMTA2-B & TraesCS4B02G306300 & $4 B(-): 595277477-595,286,089$ & 3764 & 1067 & 119.19 & 5.9095 & Nucleus & \\
\hline TaCAMTA2-D & TraesCS4D02G304500 & $4 \mathrm{D}(-): 472932312-472,941,309$ & 3988 & 1066 & 119.16 & 5.8827 & Nucleus & \\
\hline TaCAMTA3-A & TraesCS2A02G163000 & $2 A(+): 115413507-115,422,090$ & 3443 & 1026 & 113.84 & 5.7467 & Nucleus & OsCAMTA3 \\
\hline ТаСАМТАЗ-B & TraesCS2B02G188800 & $2 \mathrm{~B}(+): 164418775-164,427,330$ & 3780 & 1027 & 114.11 & 5.8397 & Nucleus & \\
\hline TaCAMTA3-D & TraesCS2D02G169900 & $2 \mathrm{D}(+): 113911362-113,919,591$ & 3783 & 1026 & 113.90 & 5.7476 & Nucleus & \\
\hline TaCAMTA4-A & TraesCS2A02G283800 & $2 A(+): 475870112-475,878,163$ & 3530 & 1027 & 114.42 & 5.2722 & Nucleus & OsCAMTA4 \\
\hline TaCAMTA4-B & TraesCS2B02G300800 & $2 B(+): 423658566-423,666,571$ & 3407 & 1028 & 114.50 & 5.1358 & Nucleus & \\
\hline TaCAMTA4-D & TraesCS2D02G282800 & $2 \mathrm{D}(+): 355676387-355,685,067$ & 3899 & 1030 & 114.91 & 5.182 & Nucleus & \\
\hline TaCAMTA5-A & TraesCS2A02G229400 & $2 A(-): 258345594-258,361,280$ & 3075 & 907 & 101.83 & 8.9645 & Nucleus & OsCAMTA5 \\
\hline TaCAMTA5-D & TraesCS2D02G237300 & 2D(-):239973233-239,987,968 & 2841 & 907 & 101.94 & 8.8824 & Nucleus & \\
\hline TaCAMTA6-B & TraesCS5B02G521100 & $5 B(-): 683109213-683,139,863$ & 3240 & 891 & 99.46 & 7.0588 & Nucleus & OsCAMTA6 \\
\hline
\end{tabular}

ID identity, Chr chromosome, ORF open reading frame, $A A$ amino acids, $p /$ isoelectric point, $M W$ molecular weight

a Chromosomal location: "+" and "-" indicate the forward and reverse strand, respectively

truncatula) with seven members $[5,11,14,15]$. The higher number of CAMTA genes may be due to gene duplication during chromosome polyploidization, since oilseed rape and soybean are tetraploid, whereas wheat is allohexaploid (AABBDD).

The subcellular locations were predicted with PlantmPLoc. According to the results, all 15 wheat CAMTA proteins were located in the nucleus, which corroborates recent studies where the CAMTA proteins have typically been located in the nucleus $[4,21]$, confirming that their main function is to regulate the expression of other genes as transcription factors.

\section{Phylogenetic analysis of the TaCAMTAs}

To investigate the phylogenetic relationships of the CAMTA gene families, a phylogenetic tree of CAMTAs from five species, including wheat, Triticum urartu, Aegilops tauschii, A. thaliana, and rice, was constructed using the neighbor-joining (NJ) algorithm. The CAMTA gene families were highly conserved during the evolution of these species (Fig. 1). All of the 36 proteins from the five species were distinctly clustered into three groups (groups A, B, and C). Seven wheat CAMTAs (TaCAMTA2-A/-B/-D, 3-A/B/D, and 6-B), one T. urartu CAMTA (TuCAMTA3), two Ae. tauschii CAMTAs (AetCAMTA2, and 3), three rice CAMTAs (OsCAMTA2, 3, and 6), and three Arabidopsis CAMTAs (AtCAMTA1, 2, and 3) were clustered into group A. In addition, six wheat CAMTAs (TaCAMTA1-A/ $-B /-D$, and TaCAMTA4-A/ $-B /-D$ ), two T. urartu CAMTAs (TuCAMTA1, and 4), two Ae. tauschii CAMTAs (AetCAMTA1 and 4), two rice
CAMTAs (OsCAMTA1, and 4), and one Arabidopsis CAMTA (AtCAMTA4) grouped into group $\mathrm{B}$, while two wheat CAMTAs (TaCAMTA5-A/-D), one T. urartu CAMTA (TuCAMTA5), one Ae. tauschii CAMTA (AetCAMTA5), one rice CAMTA (OsCAMTA5), and two Arabidopsis CAMTAs (AtCAMTA5 and 6) clustered into group C.

An unrooted phylogenetic tree was constructed using MEGA-X with the NJ algorithm and 1000 bootstrap replicates. The bootstrap values are displayed next to the branches, and the wheat CAMTAs are marked in red. The CAMTA gene ID numbers are listed as follows: A. thaliana: AtCAMTA1 (AT5G09410), AtCAMTA2 (AT5G64220), AtCAMTA3 (AT2G22300), AtCAMTA4 (AT1G67310), AtCAMTA5 (AT4G16150), AtCAMTA6 (AT3G16940); rice: OsCAMTA1 (LOC_Os01 g69910), OsCAMTA2 (LOC_Os03g09100), OsCAMTA3 (LOC_Os07g43030), OsCAMTA4 (LOC_Os04g31900), Os CAMTA5 (LOC_Os07g30774), OsCAMTA6 (LOC_Os10 g22950); T. urartu: TuCAMTA1 (TRIUR3_22499-P1), TuCAMTA3 (TRIUR3_23792-P1), TuCAMTA4 (TRIUR3_ 26386-P1), TuCAMTA5 (TRIUR3_19786-P1); Ae. tauschii: AetCAMTA1 (XP_020189402), AetCAMTA1 (XP_020179 695), AetCAMTA1 (XP_020196708), AetCAMTA1 (XP_ 020147564), and AetCAMTA1 (XP_020186933).

\section{Gene architectures and protein domain structures of the TaCAMTA members}

The number of introns in all of the 15 TaCAMTA genes varied from 10 to 13 , in which three CAMTA genes (TaCAMTA1-A/D and 6-B) possessed 10 


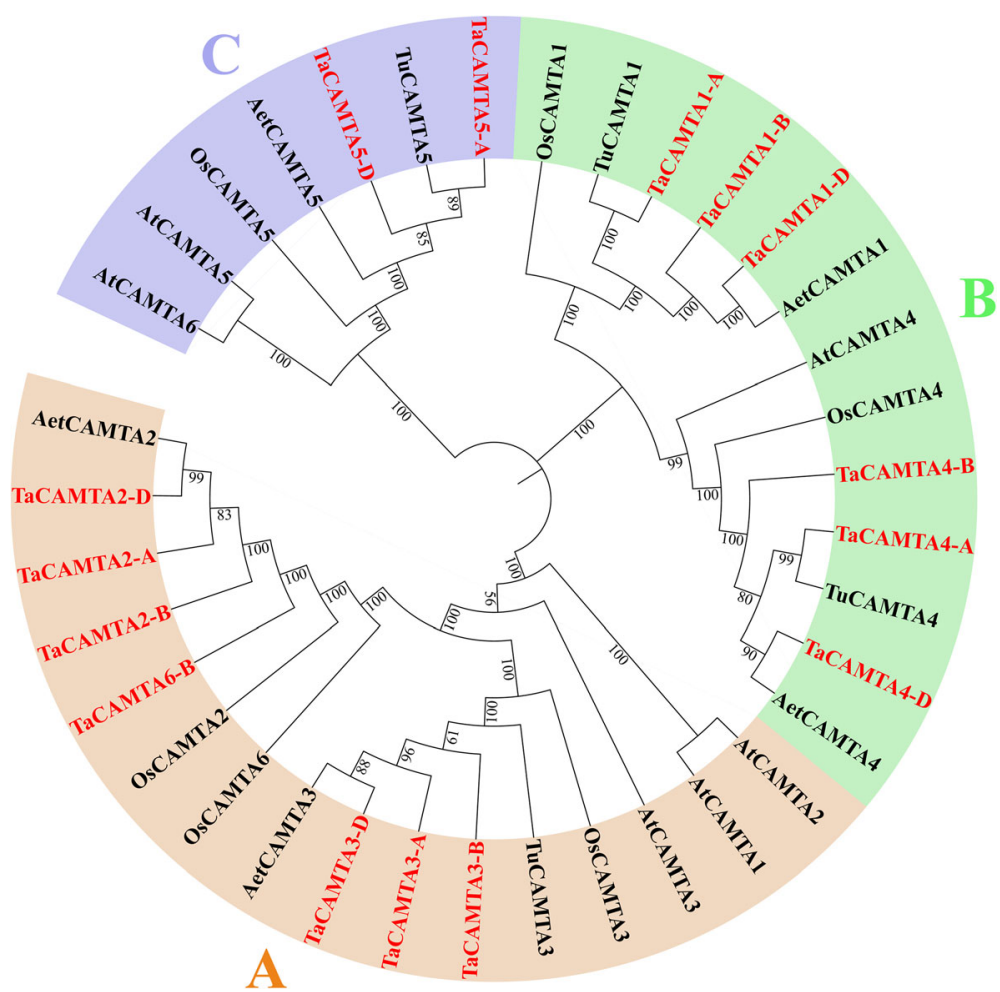

Fig. 1 Phylogenetic relationships of the CAMTA homologs in different species

introns, four CAMTA genes (TaCAMTA1-B and 4-A/ $B / D)$ possessed 11 introns, six CAMTA genes (TaCAMTA2- $A / B / D$ and $3-A / B / D)$ possessed 12 introns, and two CAMTA genes (TaCAMTA5-A/D) possessed 13 introns (Fig. 2). Similar genomic structures of the CAMTA genes have been observed in other plant species, suggesting the conservation of CAMTA genes across plant species [8, 11, 12, 21].

The exon-intron structures of the TaCAMTA genes were analyzed by comparing the coding sequences and the corresponding genomic sequences using the Gene Structure Display Server (GSDS, http://gsds.cbi.pku.edu. $\mathrm{cn} /$ ). The black box indicates exons, and the black line indicates introns.

Ten TaCAMTA proteins (TaCAMTA2-A/B/D, 3-A/B/ $\mathrm{D}, 4-\mathrm{A} / \mathrm{B} / \mathrm{D}$, and $6-\mathrm{B}$ ) were predicted to contain all of the conserved domains of a typical CAMTA protein, including a CG-1 DNA-binding domain (Pfam03859), a TIG domain involved in non-specific DNA binding (Pfam01833), several ankyrin repeats (Pfam12796), an

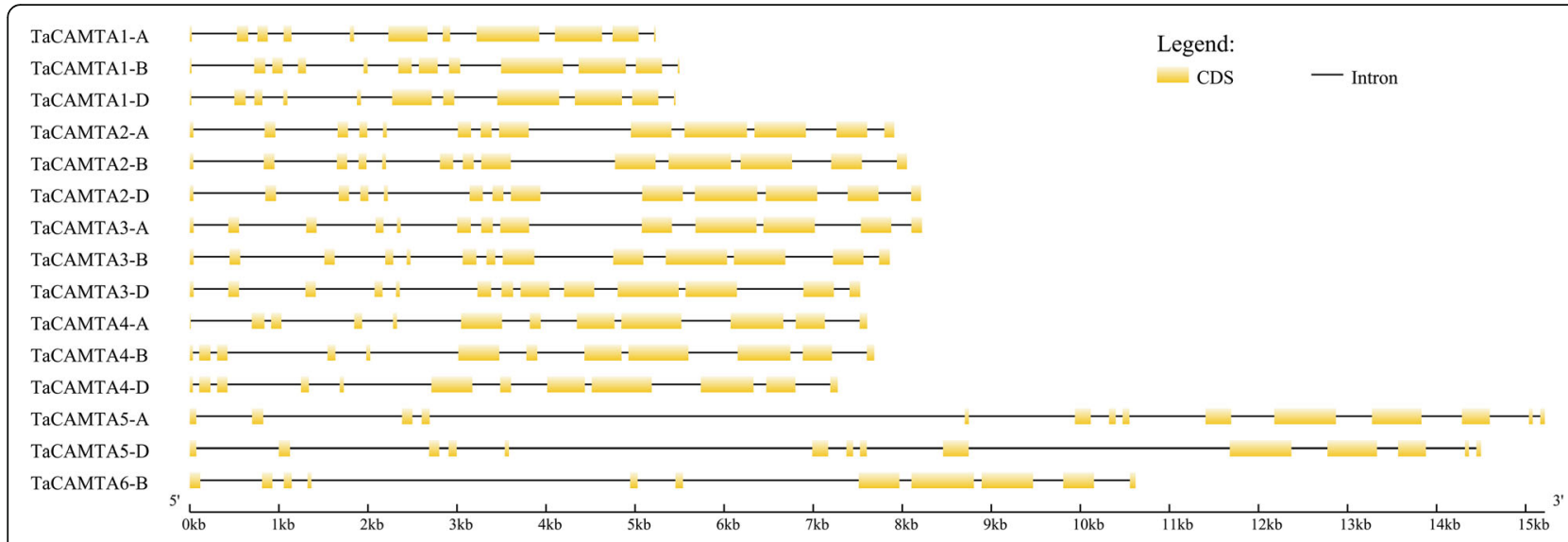

Fig. 2 Gene architectures of the TaCAMTA genes 


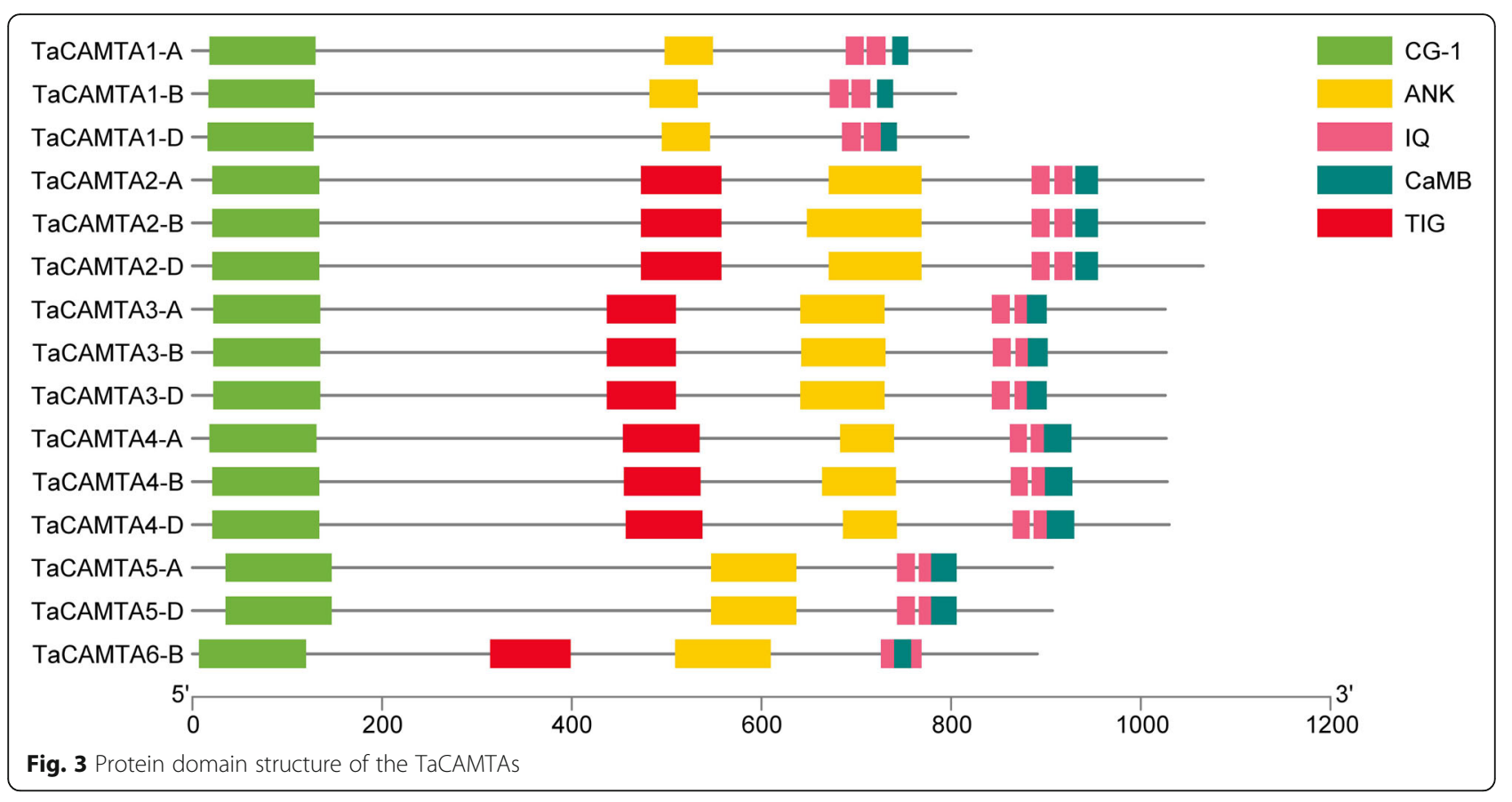

IQ motif (Pfam00612), and a calmodulin-binding domain (CaMB) (Fig. 3). Additionally, five TaCAMTA proteins (TaCAMTA1-A/B/D and TaCAMTA5-A/D) contained all of the conserved domains except for the TIG domain, which is consistent with previous studies that CAMTAs can be divided into two groups based on whether the TIG domain is present [22].

It has been confirmed that the IQ motif is able to bind with $\mathrm{CaM}$ in a $\mathrm{Ca}^{2+}$-independent manner, while the $\mathrm{CaMB}$ domain interacts with $\mathrm{CaM}$ in a $\mathrm{Ca}^{2+}$-dependent way $[5,7,8]$. It is interesting to note that all the wheat CAMTAs contain the IQ motif and a CaMB domain, indicating that wheat CAMTAs may interact with $\mathrm{CaM}$ in both a $\mathrm{Ca}^{2+}$-dependent and $\mathrm{Ca}^{2+}$-independent manner.

Analysis of the functionally conserved domains was performed using the Pfam database (http://pfam.janelia. org/) and NCBI Conserved Domains Search online tool (https://www.ncbi.nlm.nih.gov/Structure/cdd/wrpsb.cgi). CaM-binding domains (CaMBD) were analyzed in the Calmodulin Target Database (http://calcium.uhnres. utoronto.ca/ctdb/ctdb/). The domain structures of the TaCAMTAs were illustrated using TBtools software. CG-1, CG-1 DNA binding domain; TIG, TIG domain involved in non-specific DNA binding, ANK, ankyrin repeats responsible for mediating protein-protein interactions; IQ, $\mathrm{Ca}^{2+}$-independent CaM-binding IQ motifs; CaMBD, $\mathrm{Ca}^{2+}$-dependent $\mathrm{CaM}$ binding domain.

\section{Cis-acting regulatory elements in the promoters of the TaCAMTAs}

Several stresses/stimuli response cis-acting elements in the promoter regions $(2000 \mathrm{bp}$ upstream of the translation start site ATG) of the $15 \mathrm{TaCAMTA}$ genes were predicted. Seven cis-elements were used in this study: abscisic acid (ABA)-responsive element (ABRE: ACGTG, ACGTSSSC, or MACGYGB) [23], SAresponsive promoter element (SARE: TGACG) [24], environmental signal response element (G-box: CACGTG) [25], WRKY binding site (W-box: TTGAC, or TGACC/ T) [26, 27], phosphate starvation-responsive element (P1BS: GNATATNC) [28], sulfur-responsive element (SURE: GAGAC) [29], and the CAMTA binding site (CG-box: A/C/GCGCGG/T/C) [5].

The results showed that there were various known stresses/stimuli-related cis-acting elements that existed in the promoter regions of the 15 TaCAMTA genes. ABRE, SARE, W-box, and CG-box could be found in the promoter of all the $15 \mathrm{TaCAMTA}$ genes, and four TaCAMTAs (TaCAMTA1-D, 3-B, 4-A, and 4-D) contained all seven types of cis-elements in the promoter region, including ABRE, SARE, G-box, W-box, P1BS, SURE, and CG-box. Meanwhile, the remainder of the 11 TaCAMTA genes contained at least five cis-elements in their promoter region (Table 2). It has been reported that more stress-related cis-elements are located in the promoter regions of wheat CAMTA genes than other plant species $[13,14]$, indicating that wheat CAMTA genes may be more widely involved in the plant response to stress.

Tissue-specific expression patterns of the TaCAMTA genes To elucidate the possible functions of the TaCAMTA genes in wheat, qRT-PCR assay was performed to investigate the spatial expression patterns of the TaCAMTAs. 
Table 2 Numbers of stress-related cis-elements in the promoter regions of the TaCAMTA genes

\begin{tabular}{llllllll}
\hline & ABRE & SARE & G-box & W-box & P1BS & SURE & CG-box \\
\hline TaCAMTA1-A & 11 & 1 & 1 & 4 & 1 & 0 & 17 \\
TaCAMTA1-B & 4 & 3 & 0 & 5 & 1 & 1 & 11 \\
TaCAMTA1-D & 7 & 7 & 2 & 3 & 1 & 3 & 10 \\
TaCAMTA2-A & 3 & 3 & 1 & 9 & 0 & 3 & 4 \\
TaCAMTA2-B & 10 & 1 & 1 & 5 & 0 & 3 & 14 \\
TaCAMTA2-D & 6 & 2 & 1 & 9 & 0 & 3 & 8 \\
TaCAMTA3-A & 1 & 2 & 0 & 5 & 0 & 2 & 3 \\
TaCAMTA3-B & 4 & 5 & 1 & 1 & 1 & 4 & 6 \\
TaCAMTA3-D & 2 & 5 & 1 & 2 & 0 & 2 & 5 \\
TaCAMTA4-A & 9 & 2 & 1 & 8 & 1 & 5 & 9 \\
TaCAMTA4-B & 5 & 4 & 2 & 7 & 0 & 3 & 10 \\
TaCAMTA4-D & 8 & 4 & 1 & 6 & 1 & 4 & 10 \\
TaCAMTA5-A & 1 & 1 & 0 & 7 & 0 & 3 & 5 \\
TaCAMTA5-D & 1 & 3 & 1 & 6 & 0 & 1 & 3 \\
TaCAMTA6-B & 1 & 1 & 0 & 11 & 1 & 2 & 2
\end{tabular}

ABRE ABA-responsive element, SARE SA-responsive promoter element, G-box environmental signal response element, $W$-box WRKY binding site, P1BS phosphate starvation-responsive element, SURE sulfur-responsive element, $C G$ box the CAMTA binding site

The results showed that all of the 15 TaCAMTA genes were expressed in multiple tissues with different expression levels. TaCAMTA3-D, 5-A, and 5-D showed highest expression level in shoot during seedling stage, while highest expression level of TaCAMTA1-D and 3-B was observed in spike during reproductive stage, suggesting that various CAMTA gene members maintain different functions in wheat growth and development (Fig. 4).

Expression of TaCAMTAs were analyzed by qRT-PCR in root and shoot of ten-day-old seedlings, root, stem, leaf, spike at flowering in reproductive stage, and grain 15 DAA (days after athesis). The relative expression levels were normalized to 1 in roots of ten-day-old seedlings $(0 \mathrm{~h})$.

\section{Expression profiles of the TaCAMTA genes during abiotic stress}

Previous studies have shown that plant CAMTAs could be involved in diverse environmental stresses. AtCAMTA1 and SISR1L played a positive function in drought stress in Arabidopsis and tomato $[18,30]$, while plant CAMTAs also respond to salt and cold stress $[11,16,31]$. However, to date there is no information available on wheat CAMTAs involved in abiotic stresses. In this light, the expression profiles of the TaCAMTAs were analyzed under drought, $\mathrm{NaCl}$, cold and heat stress. Under drought stress, TaCAMTA1-A, 1-B, $1-D, 2-B, 4-B, 4-D, 5-A, 5-D$ and $6-B$ were significantly upregulated, while the expressions of TaCAMTA2-A, 2-D, 3-A, 3- $D$ were moderately down-regulated (Fig. $5 \mathrm{a}$ ). In response to $\mathrm{NaCl}$ stress, the expressions of TaCAMTA1-A, 1-D, 5- $A$,
5- $D$ and 6-B were enhanced, while the expressions of TaCAMTA2-A, 2-B, 2-D, 3-A, 3-B, 4-A, 4-B, 4-D were inhibited (Fig. $5 \mathrm{~b}$ ). In the cold treatment assay, the expressions of TaCAMTA1-A, 1-D, 3-A, and 3-D increased dramatically, while the expressions of TaCAMTA2- $A$, 4- $A$, 4- $B$, and $4-D$ decreased (Fig. $5 \mathrm{C}$ ). In the heat treatment group, the expressions of TaCAMTA1- $A, 1-B, 1-D, 2-A$, and 4- $B$ remarkably increased within one hour; by contrast, the expressions of TaCAMTA2-B, 2-D, 3-B, 4- $A, 5-A, 5-D$, and 6- $B$ were repressed, especially in the late stage of heat treatment (Fig. 5d).

It can been found that the expression of each TaCAMTA gene could respond to at least one abiotic stress, and TaCAMTA1-A and 1-D could be upregulated by all abiotic stresses used in this study, including drought, $\mathrm{NaCl}$, cold and heat stress (Fig. 5), implying different regulations and functions of TaCAMTA gene members while coping with various abiotic stresses in wheat. It can also been found that the CAMTA genes from same homoeologous group showed similar expression patterns, such as TaCAMTA1-A/B/D under drought treatment (Fig. 5a), TaCAMTA5-A/D under $\mathrm{NaCl}$ treatment (Fig. 5b), and TaCAMTA1-A/B/D under heat shock stress (Fig. 5d). However, several homoeologous CAMTA genes from same group showed different expression patterns under stresses. For example, TaCAMTA1-A/D and TaCAMTA3-A/D were upregulated by cold treatment, while the expressions of TaCAMTA1-B and TaCAMTA3-B were relatively stable (Fig. 5c). These results suggest that the homoeologous CAMTA genes from the same group generally have the same regulations and functions, while functional differentiation may have occurred in some homoeologous CAMTA genes.

Expression of TaCAMTAs were analyzed by qRT-PCR in roots of ten-day-old seedlings, which had been treated with 16.1\% PEG 6000 (drought), $200 \mathrm{mM} \mathrm{NaCl}, 4{ }^{\circ} \mathrm{C}$ (cold) and $40{ }^{\circ} \mathrm{C}$ (heat) for indicated durations. The relative expression levels were normalized to 1 in unstressed plants $(0 \mathrm{~h})$.

\section{Prediction of target genes by CAMTA}

It has been found that CAMTA has the specific binding activity to CGCG box in promoter of target genes [5]. In this study, a search of the data base revealed that cis-acting elements ACGCGG/CCGCGT were present in the promoter regions of about 584 genes (more than two copies) in wheat genome, which were considered as potential target genes by CAMTA (Additional file 1:Table $\mathrm{S} 1$ ). These genes are related to RNA regulation (69 genes), protein degradation (42 genes), signalling transduction (30 genes), biotic and abiotic stresses (17 genes), hormone metabolism (17 genes), and lipid metabolism (13 genes), demonstrating that CAMTA can be widely 


\begin{tabular}{|c|c|c|c|c|c|c|c|c|}
\hline & \multicolumn{2}{|c|}{ Seedling } & \multicolumn{5}{|c|}{ Reproductive Stage } & \\
\hline & Root & Shoot & Root & Stem & Leaf & Spike & Grain & \\
\hline TaCAMTA1-A & 1.00 & 0.51 & 0.21 & 0.36 & 0.39 & 0.65 & 0.33 & \\
\hline TaCAMTA1-B & 1.00 & 0.51 & 0.25 & 1.48 & 0.37 & 1.19 & 0.07 & \\
\hline TaCAMTA1-D & 1.00 & 1.01 & 0.13 & 1.05 & 0.42 & 2.20 & 0.43 & \\
\hline TaCAMTA2-A & 1.00 & 0.52 & 0.28 & 0.26 & 0.17 & 0.22 & 1.03 & \\
\hline TaCAMTA2-B & 1.00 & 0.97 & 0.24 & 0.40 & 0.60 & 0.20 & 0.17 & 350 \\
\hline TaCAMTA2-D & 1.00 & 0.84 & 0.29 & 0.31 & 0.82 & 0.30 & 0.46 & 3.00 \\
\hline TaCAMTA3-A & 1.00 & 0.77 & 0.09 & 0.64 & 0.30 & 0.95 & 0.80 & 2.50 \\
\hline TaCAMTA3-B & 1.00 & 1.64 & 0.44 & 0.61 & 1.83 & 1.93 & 1.51 & $\begin{array}{l}2.00 \\
1.50\end{array}$ \\
\hline TaCAMTA3-D & 1.00 & 1.85 & 0.37 & 0.59 & 0.94 & 0.64 & 0.51 & 1.00 \\
\hline TaCAMTA4- $A$ & 1.00 & 0.85 & 0.22 & 0.30 & 0.47 & 0.21 & 0.21 & 0.50 \\
\hline TaCAMTA4-B & 1.00 & 0.41 & 0.12 & 0.20 & 0.33 & 0.89 & 0.45 & 0.00 \\
\hline TaCAMTA4-D & 1.00 & 0.46 & 0.20 & 0.27 & 0.23 & 0.14 & 0.08 & \\
\hline TaCAMTA5- $A$ & 1.00 & 3.08 & 0.37 & 1.19 & 1.93 & 1.22 & 0.90 & \\
\hline TaCAMTA5-D & 1.00 & 3.28 & 0.33 & 1.17 & 1.89 & 1.47 & 1.02 & \\
\hline ТаCAMTA6-B & 1.00 & 1.10 & 0.20 & 0.31 & 0.65 & 0.16 & 0.08 & \\
\hline
\end{tabular}

Fig. 4 Expression patterns of the TaCAMTA genes in multiple tissues

involved in plant development and growth, as well as coping with stresses.

\section{Conclusions}

In conclusion, 15 CAMTA genes were identified in wheat in the present study. Analysis of the gene structure and protein domain, physicochemical properties, and the phylogenetic relationships indicated that the CAMTA gene family was highly conserved during plant evolution. Tissue-specific expression analysis showed that all of the 15 TaCAMTA genes were expressed in multiple tissues with different expression levels, suggesting that various CAMTA gene members maintain different functions in wheat growth and development. Under abiotic stress, the expressions of all the TaCAMTA genes could respond to at least one abiotic stress, implying different regulations and functions of TaCAMTA gene members while coping with various abiotic stresses in wheat. 584 genes in wheat genome were predicted to be potential target genes by CAMTA, demonstrating that CAMTA can be widely involved in plant development and growth, as well as coping with stresses. Our findings provide new insight into the CAMTA gene family in wheat as well as a foundation for further studies on the roles of TaCAMTA genes in wheat development and growth as well as the stress response.

\section{Methods}

Genome-wide identification of the CAMTA gene family

Protein sequences of Triticum aestivum (IWGSC1.1), Triticum urartu (ASM34745v1), and Aegilops tauschii (ASM34733v1) were obtained from the Ensemble plant database (http://plants.ensembl.org) to predict the CAMTA genes [32]. The Hidden Markov Model (HMM) profile of the CG-1 domain (PF03859), the ANK repeat domain (PF00023), and the IQ domain (PF00612) sequences were downloaded from the PFAM database [33] and used to examine all wheat protein sequences using the HMMER search tool with E-value $<=0.0001$ [34]. The obtained protein sequences were checked using the National Center for Biotechnology Information (NCBI) Conserved domain database (CDD) search (https://www. ncbi.nlm.nih.gov/cdd) to identify the conserved protein domain with the default parameters. The redundant sequences containing complete CG-1, ANK repeats, and the IQ domain were further removed by alignment, and the remainder were considered as putative CAMTA genes. Finally, the biochemical parameters of the TaCAMTA proteins were calculated using the Compute $\mathrm{pI} / \mathrm{MW}$ tool in 


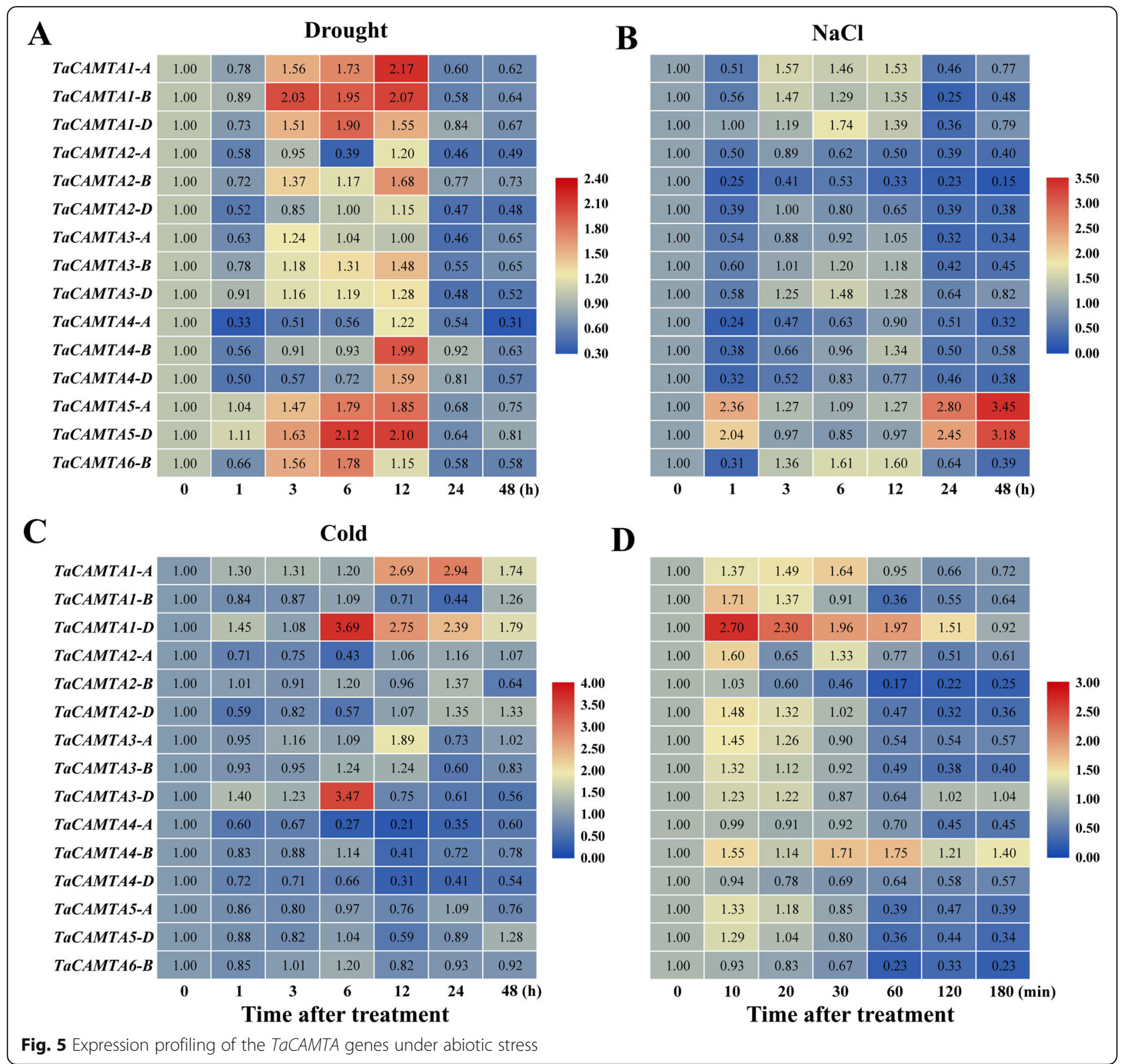

the ExPASy database with the default parameters (https:// web.expasy.org/compute_pi/). Subcellular localization of the TaCAMTA proteins was predicted online by PlantmPLoc with the default parameters (http://www.csbio.sjtu. edu.cn/bioinf/plant-multi/) [35].

\section{Phylogenetic tree construction and sequence analysis}

Protein sequences from Arabidopsis and rice were obtained from NCBI (http://www.ncbi.nlm.nih.gov/) and Ensembl Plants (http://plants.ensembl.org/index.html). The amino acid sequences of all CAMTAs were aligned using the ClustalX program with the default parameters, and a phylogenetic tree was constructed in MEGA-X using the neighbor-joining method with 1000 bootstrap replicates
[36]. The display of the phylogenetic tree was optimized using the Interactive Tree Of Life (iTOL) v4 [37].

The schematic structures of the TaCAMTA genes were analyzed online using the Gene Structure Display Server 2.0 based on exon/intron data (GSDS 2.0, http:// gsds.cbi.pku.edu.cn/) [38]. The domain structures of the TaCAMTA proteins were analyzed in the Pfam database (http://pfam.janelia.org/) and NCBI Conserved Domains Search online tool against database CDD v3.18 with Evalue threshold $<=0.01$ (https://www.ncbi.nlm.nih.gov/ Structure/cdd/wrpsb.cgi) [39]. The CaMB domain was specifically analyzed using Calmodulin Binding Site Search in the Calmodulin Target Database (http://calcium.uhnres.utoronto.ca/ctdb/ctdb/). 
Prediction of cis-acting elements in the TaCAMTA genes To investigate the cis-elements in the promoter sequences of the TaCAMTA genes, 2-kb sequences upstream of the initiation codon (ATG) were collected from the Ensembl plant database (http://plants.ensembl.org) and subjected to the PLACE database with the default parameters (https://www.dna.affrc.go.jp/PLACE) [32, 40].

\section{Plant materials and treatments}

Jinhe 9123, a wheat variety cultivated by our lab, was used in this study. For stress treatment experiments, seeds were surface-sterilized in $1 \% \mathrm{NaOCl}$ and repeatedly rinsed with tap water, then seeded in $1 / 2$ Hoagland nutrient solution after immersion and imbibition for 12 $\mathrm{h}$ [41], and hydroponically cultivated in the incubator with a $16 / 8$-h photoperiod at $25^{\circ} \mathrm{C}$. Four ten-day-old homogeneous seedlings groups, each of which included 30 seedlings from three biological replicates, were subjected to different treatments, including 16.1\% PEG6000, $200 \mathrm{mM} \mathrm{NaCl}$ and cold $\left(4{ }^{\circ} \mathrm{C}\right)$ for $1,3,6,12,24$ and 48 $\mathrm{h}$, and heat $\left(40^{\circ} \mathrm{C}\right)$ for $10,20,30,60,120,180 \mathrm{~min}$. The shoot tissue was sampled for the experiment. For analyses of tissue-specific expression patterns, root and shoot from ten-day-old seedlings, root, stem, leaf, spike at flowering in reproductive stage, and grain 15 DAA were collected from wheat plants. Collected samples were immediately frozen in liquid nitrogen and stored at $-80^{\circ} \mathrm{C}$ for RNA extraction.

\section{RNA isolation and gene expression analysis}

Total RNA of collected samples was isolated using the EasyPure ${ }^{\circ}$ Plant RNA Kit (ER301-01, Transgen, China). For reverse transcription, the first-strand cDNA was synthesized using a PrimeScript ${ }^{\mathrm{Tw}}$ RT reagent Kit (RR047A, TaKaRa, Japan). Quantitative real-time PCR (qRT-PCR) for examination of the TaCAMTAs expression patterns were performed using the TB Green ${ }^{\text {Tx }}$ Premix Ex Taq ${ }^{\text {Tix }}$ II (RR820A, TaKaRa, Japan) with 7500 Real-Time PCR System (Applied Biosystem, USA). Gene specific and internal reference gene TaActin primers were listed in Additional file 2: Table S2. The qRT-PCR program was carried out as follows: predenaturation at $95^{\circ} \mathrm{C}$ for $30 \mathrm{~s}$; denaturation at $95^{\circ} \mathrm{C}$ for $5 \mathrm{~s}$, annealing at $58^{\circ} \mathrm{C}$ for $30 \mathrm{~s}$, extension at $60^{\circ} \mathrm{C}$ for $34 \mathrm{~s}, 45$ cycles. $2^{-\Delta \Delta \mathrm{Ct}}$ method was used to analyze the data [42]. All experiments were performed with three technical replicates and three biological replicates, and the data were represented by mean value of three biological replicates.

\section{Prediction of target genes by CAMTA}

Prediction of the target genes by CAMTA were performed as described by Yang and Poovaiah (2002) with some modifications [5]. 1-kb sequences upstream of the initiation codon (ATG) of all genes in wheat genome were collected as promoter sequences, and a search of cis-acting elements ACGCGG/CCGCGT (CGCG box) were conducted. The genes with more than two copies of CGCG box were considered as potential target genes by CAMTA. The MapMan tool was used to facilitate the assignment of different gene sets into functional categories (BINs). A MapMan mapping file that mapped the genes into BINs via hierarchical ontologies through the searching of a variety of reference databases was generated using the Mercator tool (http://mapman.gabipd. org/web/guest/app/mercator) [43].

\section{Supplementary information}

Supplementary information accompanies this paper at https://doi.org/10. 1186/s12863-020-00916-5.

Additional file 1: Table S1. Predicted target genes by CAMTA.

Additional file 2: Table S2. Primer sequences of TaCAMTA and TaActin genes used for qRT-PCR analysis.

\section{Abbreviations}

CAMTA: Calmodulin-binding transcription activator;; CaM: Calmodulin; CML: Calmodulin-like proteins; CDPK: Calcium-dependent protein kinases; CBL: Calcineurin B-like proteins; CaMBP: Calmodulin-binding proteins; TIG: A transcription factor immunoglobulin-like DNA-binding domain; ANK: Ankyrin repeats; CaMBD: Calmodulin-binding domain; CBF2: C-repeat binding factor2; RD26: Responsive to dehydration26; ERD7: Early response to dehydration7; RAB18: Responsive to ABA18; LTPs: Lipid transfer proteins; COR78: Coldregulated78; HSPS: Heat shock proteins; EDS1: Enhanced disease susceptibility1; SA: Salicylic acid; VIGS: Virus-induced gene silencing; NJ: Neighbor-joining; ABA: Abscisic acid; ABRE: ABA-responsive element; SARE: SA-responsive promoter element; P1BS: Phosphate starvationresponsive element; SURE: Sulfur-responsive element; DAA: Days after athesis; HMM: Hidden Markov Model; NCBI: National Center for Biotechnology Information; CDD: Conserved domain database; iTOL: Interactive Tree Of Life; GSDS: Gene Structure Display Server; qRT-PCR: Quantitative real-time PCR

\section{Acknowledgments}

We would like to thank Dr. Xin-ye Liu of Institute of Molecular Cell Biology, School of Life Sciences, Hebei Normal University, Dr. Yong-qiang Wang of Institute of Cotton, Hebei Academy of Agriculture and Forestry Sciences, and Dr. Chuang-qi Wei of Institute of Genetics and Physiology, Hebei Academy of Agriculture and Forestry Sciences for their help in prediction and functional annotation of target genes.

\section{Authors' contributions}

ZS designed the study and wrote the manuscript. YF carried out bioinformation analyses. HFH and DFS carried out the qRT-PCR analyses. LYW and LMY collected plant materials. ZH and CJF carried out RNA isolation. All authors have read and approved the final manuscript.

\section{Funding}

This research was financially supported by the National Key Research and Development Program of China (2016YFD0101802), the National Natural Science Foundation of China (31600216), the Natural Science Foundation of Hebei Province, China (C2017301066), and the HAAFS Agriculture Science and Technology Innovation Project (2019-4-8-1). The funding bodies played no role in the design of the study and collection, analysis, and interpretation of data and in writing the manuscript.

Availability of data and materials

The data sets supporting the article are included within the article and its additional files.

Ethics approval and consent to participate Not applicable. 


\section{Consent for publication}

Not applicable.

\section{Competing interests}

The authors declare that they have no competing interests.

\section{Author details}

'Institute of Genetics and Physiology, Hebei Academy of Agriculture and Forestry Sciences/Plant Genetic Engineering Center of Hebei Province, Shijiazhuang 050051, People's Republic of China. ${ }^{2}$ Agriculture and Rural Bureau of Nanhe County, Xingtai 054400, People's Republic of China.

Received: 30 January 2020 Accepted: 6 September 2020 Published online: 14 September 2020

\section{References}

1. Dodd AN, Kudla J, Sanders D. The language of calcium signaling. Annu Rev Plant Biol. 2010;61:593-620

2. Hashimoto K, Kudla J. Calcium decoding mechanisms in plants. Biochimie. 2011:3:2054-9.

3. Abbas N, Maurya JP, Senapati D, Gangappa SN, Chattopadhyay S. Arabidopsis CAM7 and HY5 physically interact and directly bind to the HY5 promoter to regulate its expression and thereby promote photomorphogenesis. Plant Cell. 2014;26:1036-52.

4. Wei M, Xu X, Li C. Identification and expression of CAMTA genes in Populus trichocarpa under biotic and abiotic stress. Sci Rep. 2017:7:17910.

5. Yang T, Poovaiah BW. A calmodulin-binding/CGCG box DNA-binding protein family involved in multiple signaling pathways in plants. J Bio Chem. 2002:277:45049-58

6. Finkler A, Ashery-Padan R, Fromm H. CAMTAs: calmodulin-binding transcription activators from plants to human. FEBS Lett. 2007:581:3893-8.

7. Bouche N, Scharlat A, Snedden W, Bouchez D, Fromm H. A novel family of calmodulin-binding transcription activators in multicellular organisms. J Biol Chem. 2002;277:21851-61.

8. Yang TB, Peng H, Whitaker BD, Conway WS. Characterization of a calcium/ calmodulin regulated SR/CAMTA gene family during tomato fruit development and ripening. BMC Plant Biol. 2012:12:19.

9. Choi MS, Kim MC, Yoo JH, Moon BC, Koo SC, Park BO, et al. Isolation of a calmodulin-binding transcription factor from rice (Oryza sativa L.). J Biol Chem. 2005;280:40820-31.

10. Shangguan L, Wang X, Leng X, Liu D, Ren G, Tao R, et al. Identification and bioinformatic analysis of signal responsive/calmodulin-binding transcription activators gene models in Vitis vinifera. Mol Biol Rep. 2014;41:2937-49.

11. Yue R, Lu C, Sun T, Peng T, Han X, Oi J, et al. Identification and expression profiling analysis of calmodulin-binding transcription activator genes in maize (Zea mays L.) under abiotic and biotic stresses. Front Plant Sci. 2015:6:576.

12. Wang G, Zeng H, Hu X, Zhu Y, Chen Y, Shen C, et al. Identification and expression analyses of calmodulin-binding transcription activator genes in soybean. Plant Soil. 2015;386:205-21.

13. Rahman $H$, Xu YP, Zhang XR, Cai XZ. Brassica napus genome possesses extraordinary high number of CAMTA genes and CAMTA3 contributes to PAMP triggered immunity and resistance to Sclerotinia sclerotiorum. Front Plant Sci. 2016:7:581.

14. Yang $Y$, Sun $T$, Xu L, Pi E, Wang S, Wang H, Shen C. Genome-wide identification of CAMTA gene family members in Medicago truncatula and their expression during root nodule symbiosis and hormone treatments. Front Plant Sci. 2015;6:459.

15. Zhang J, Pan X, Ge T, Yi S, Lv Q, Zheng Y, et al. Genome-wide identification of citrus CAMTA genes and their expression analysis under stress and hormone treatments. J Hortic Sci Biotechnol. 2018;94:331-40.

16. Kim Y, Park S, Gilmour SJ, Thomashow MF. Roles of CAMTA transcription factors and salicylic acid in configuring the low-temperature transcriptome and freezing tolerance of Arabidopsis. Plant J. 2013;75:364-76.

17. Doherty CJ, Van Buskirk HA, Myers SJ, Thomashow MF. Roles for Arabidopsis CAMTA transcription factors in cold-regulated gene expression and freezing tolerance. Plant Cell. 2009:21:972-84.

18. Pandey N, Ranjan A, Pant P, Tripathi RK, Ateek F, Pandey HP, et al. CAMTA regulates drought responses in Arabidopsis thaliana. BMC Genomics. 2013;14:216.

19. Du L, Ali GS, Simons KA, Hou J, Yang T, Reddy AS, Poovaiah BW. Ca ${ }^{(2+)} /$ calmodulin regulates salicylic-acid-mediated plant immunity. Nature. 2009; 457:1154-8.
20. Wang Y, Wei F, Zhou H, Liu N, Niu X, Yan C, et al. TaCAMTA4, a calmodulininteracting protein, involved in defense response of wheat to Puccinia triticina. Sci Rep. 2019;9:641.

21. Pant P, labal Z, Pandey BK, Sawant SV. Genome-wide comparative and evolutionary analysis of calmodulin-binding transcription activator (CAMTA) family in Gossypium species. Sci Rep. 2018;8:5573.

22. Rahman H, Yang J, Xu YP, Munyampundu JP, Cai XZ. Phylogeny of plant CAMTAs and role of AtCAMTAs in nonhost resistance to Xanthomonas oryzae pv oryzae. Front Plant Sci. 2016;7:177.

23. Kaplan B, Davydov O, Knight H, Galon Y, Knight MR, Fluhr R, Fromm H. Rapid transcriptome changes induced by cytosolic $\mathrm{Ca}^{2+}$ transients reveal ABRE-related sequences as $\mathrm{Ca}^{2+}$-responsive cis elements in Arabidopsis. Plant Cell. 2006;18:2733-48

24. Després C, Chubak C, Rochon A, Clark R, Bethune T, Desveaux D, Fobert PR. The Arabidopsis NPR1 disease resistance protein is a novel cofactor that confers redox regulation of DNA binding activity to the basic domain/ leucine zipper transcription factor TGA1. Plant Cell. 2003;15:2181-91.

25. Chakravarthy S, Tuori RP, D'Ascenzo MD, Fobert PR, Despres C, Martin GB. The tomato transcription factor Pti4 regulates defense-related gene expression via GCC box and non-GCC box cis elements. Plant Cell. 2003;15:3033-50.

26. Nishiuchi T, Shinshi H, Suzuki K. Rapid and transient activation of transcription of the ERF3 gene by wounding in tobacco leaves: possible involvement of NtWRKYs and autorepression. J Biol Chem. 2004;279:55355-61.

27. Yu DQ, Chen $\mathrm{CH}$, Chen ZX. Evidence for an important role of WRKY DNA binding proteins in the regulation of NPR1 gene expression. Plant Cell. 2001; 13:1527-39.

28. Schunmann PH, Richardson AE, Smith FW, Delhaize E. Characterization of promoter expression patterns derived from the Pht1 phosphate transporter genes of barley (Hordeum vulgare L.). J Exp Bot. 2004;55:855-65.

29. Maruyama-Nakashita A, Nakamura Y, Watanabe-Takahashi A, Inoue E, Yamaya $\mathrm{T}$, Takahashi $\mathrm{H}$. Identification of a novel cis-acting element conferring sulfur deficiency response in Arabidopsis roots. Plant J. 2005;42:305-14.

30. Li XH, Huang L, Zhang YF, Ouyang ZG, Hong YB, Zhang HJ, et al. Tomato SR/CAMTA transcription factors SISR1 and SISR3L negatively regulate disease resistance response and SISR1L positively modulates drought stress tolerance. BMC Plant Biol. 2014;14:286.

31. Buyuk I, Ihan E, Sener D, Ozsoy AU, Aras S. Genome-wide identification of CAMTA gene family members in Phaseolus vulgaris $\mathrm{L}$. and their expression profiling during salt stress. Mol Biol Rep. 2019;46:2721-32.

32. Kersey PJ, Allen JE, Armean I, Boddu S, Bolt BJ, Carvalho-Silva D, et al. Ensembl genomes 2016: more genomes, more complexity. Nucleic Acids Res. 2016:44:574-80.

33. Finn RD, Mistry J, Schuster-Bockler B, Griffiths-Jones S, Hollich V, Lassmann T, et al. Pfam: clans, web tools and services. Nucleic Acids Res. 2006:34:247-51.

34. Wheeler TJ, Eddy SR. Nhmmer: DNA homology search with profile HMMs. Bioinformatics. 2013;29:2487-9.

35. Chou KC, Shen HB. Plant-mPLoc: a top-down strategy to augment the power for predicting plant protein subcellular localization. PLoS One. 2010:5:e11335.

36. Kumar S, Stecher G, Li M, Knyaz C, Tamura K. Molecular evolutionary genetics analysis across computing platforms. Mol Biol Evol. 2018:35:1547-9.

37. Letunic I, Bork P. Interactive tree of life (iTOL) v4: recent updates and new developments. Nucleic Acids Res. 2019;47:256-9.

38. Hu B, Jin J, Guo AY, Zhang H, Luo J, Gao G. GSDS 2.0: an upgraded gene feature visualization server. Bioinformatics. 2015;31:1296-7.

39. Marchler-Bauer A, Bo Y, Han L, He J, Lanczycki CJ, Lu S, et al. CDD/SPARCLE: functional classification of proteins via subfamily domain architectures. Nucleic Acids Res. 2017:45:200-3.

40. Higo K, Ugawa Y, Iwamoto M, Higo H. PLACE: a database of plant cis-acting regulatory DNA elements. Nucleic Acids Res. 1998:26:358-9.

41. Hoagland DR, Arnon DI. The water culture method for growing plants without soil. Calif Agric Exp Sta Circ. 1950:347:32.

42. Livak KJ, Schmittgen TD. Analysis of relative gene expression data using real-time quantitative PCR and the $2^{-\Delta \Delta C T}$ method. Methods. 2001;25:402-8.

43. Thimm O, Bläsing $O$, Gibon $Y$, Nagel A, Meyer S, Krüger $P$, Selbig J, Müller LA, Rhee SY, Stitt M. MAPMAN: a user-driven tool to display genomics datasets onto diagrams of metabolic pathways and other biological processes. Plant J. 2004;37:914-39.

\section{Publisher's Note}

Springer Nature remains neutral with regard to jurisdictional claims in published maps and institutional affiliations. 